PROCEEDINGS OF THE

AMERICAN MATHEMATICAL SOCIETY

Volume 132, Number 5, Pages 1531-1541

S 0002-9939(03)07389-1

Article electronically published on December 23, 2003

\title{
REGULARITY, PARTIAL ELIMINATION IDEALS AND THE CANONICAL BUNDLE
}

\author{
MATTHEW G. JONES
}

(Communicated by Michael Stillman)

\begin{abstract}
We present partial elimination ideals, which set-theoretically cut out the multiple point loci of a generic projection of a projective variety, as a way to bound the regularity of a variety in projective space. To do this, we utilize a combination of initial ideal methods and geometric methods. We first define partial elimination ideals and establish through initial ideal methods the way in which, for a given ideal, the regularity of the partial elimination ideals bounds the regularity of the given ideal. Then we explore the partial elimination ideals as a way to compute the canonical bundle of the generic projection of a variety and the canonical bundles of the multiple point loci of the projection, and we use Kodaira Vanishing to bound the regularity of the partial elimination ideals.
\end{abstract}

\section{BACKGROUND}

1.1. Regularity, initial and generic initial ideals. In this section, we review the key results and definitions needed for the proof in section 2. None of the results in this section are new, and a good place to start for proofs not presented here is Gre98. We will work exclusively over the complex numbers $\mathbf{C}$ throughout this paper.

Note 1.1. A multiplicative monomial order is a refinement of the degree order and the variable order $x_{0}<x_{1}<x_{2}<\ldots<x_{r}$ that respects multiplication. The most common orders are the lexicographic order, abbreviated lex, and the reverse lexicographic order, abbreviated rlex. In addition, we will have need of the h-elimination order: for our purposes, this is an amalgamation of the first two orders, and in other literature may also be called the $\{\mathbf{h}, \mathbf{r}+\mathbf{1}-\mathbf{h}\}$-product order. The $h$-elimination order uses the lex order on the first $h$ variables, and breaks lexicographic ties by using rlex on the remaining variables.

We let $U=\left(u_{0}, u_{1}, \ldots, u_{r}\right), u_{i} \in \mathbf{Z}$, and write $x^{U}$ for $a^{u_{0}} x_{1}^{u_{1}} \cdots x_{r}^{u_{r}}$.

Received by the editors October 5, 2001 and, in revised form, January 22, 2003.

2000 Mathematics Subject Classification. Primary 51N15.

The bulk of this work was completed under the direction of Mark Green as part of my Ph.D. thesis at UCLA. I am very grateful to Professor Green for all his guidance, the time and energy he devoted to me and the knowledge he imparted upon me. I am also grateful to the UCLA Mathematics Department for its support. 
Definition 1.2. For a fixed monomial order and a homogeneous polynomial $F$, write

$$
F=\sum c_{U} x^{U}
$$

$c_{U} \in \mathbf{C}$ nonzero. We define the initial term of $F, \operatorname{in}(F)=c_{V} x^{V}$, where $c_{V} x^{V}$ is a nonzero monomial appearing in the expression for $F$ above, and $x^{V}>x^{U}$ for every other $U$ appearing in (1). We also have the lead term of $F, \operatorname{LeadTerm}(F)=x^{V}$. The initial ideal of an ideal $I$, written $\operatorname{in}(I)$, is generated by $\{\operatorname{in}(F) \mid F \in I\}$.

Definition 1.3. An elementary move $e_{k}$, for $0 \leq k \leq r-1$, is defined by $e_{k}\left(x^{U}\right)=x^{\hat{U}}$, where $\hat{U}=\left(u_{0}, \ldots, u_{k-1}, u_{k}+1, u_{k+1}-1, u_{k+1}, \ldots, u_{r}\right)$, and we say $x^{U}=0$ if some $u_{i}<0$.

Proposition 1.4. The following are equivalent for a monomial ideal I:

(1) ( $I$ is closed under elementary moves.) If $x^{U} \in I$, then for every elementary move $e_{k}, e_{k}\left(x^{U}\right) \in I$.

(2) $g(I)=I$ for every $g$ belonging to the Borel subgroup $B$.

(3) $\operatorname{in}(g(I))=I$ for every $g$ in some open neighborhood of the identity in $B$.

Definition 1.5. A monomial ideal $I$ is Borel-fixed if any (hence all) of the conditions of the proposition hold for $I$.

We collect several needed but well-known results into the following.

\section{Theorem 1.6.}

(1) Galligo's Theorem. For any multiplicative monomial order and any homogeneous ideal $I$, there is a Zariski open subset $W \subset G L$ such that in $(g(I))$ is constant and Borel-fixed for $g \in W$. Galligo's theorem allows us to define, for a homogeneous ideal $I$, the generic initial ideal of $I, \operatorname{gin}(I)=\operatorname{in}(g(I))$ for $g \in W$.

(2) For a Borel-fixed monomial ideal $I,\left(I: x_{r}\right)=(I: m)$.

(3) For a Borel-fixed monomial ideal $I$, sat $(I)$ is the degree of the largest generator of I involving $x_{r}$. In particular, $I$ is saturated if no generator of I involves $x_{r}$.

(4) For a Borel-fixed monomial ideal $I, \operatorname{reg}(I)$ is the maximal degree of a generator of $I$.

(5) For a homogeneous ideal I, using any order,

$$
\operatorname{reg}(I) \leq \operatorname{reg}(\operatorname{in}(I)) .
$$

In particular, $\operatorname{reg}(I)$ is bounded by the maximal degree of a generator of $\operatorname{gin}(I)$.

(6) Bayer-Stillman BS87. For a homogeneous ideal I, using the rlex order, $\operatorname{sat}(I)=\operatorname{sat}(\operatorname{gin}(I))$, which is in turn equal to the maximal degree of a generator of $\operatorname{gin}(I)$ involving $x_{r}$. In particular, $I$ is saturated if and only if no generator of the rlex $\operatorname{gin}(I)$ involves $x_{r}$. The regularity of $I$ is equal to the regularity of the rlex $\operatorname{gin}(I)$, or equivalently to the maximal degree of a generator of the rlex $\operatorname{gin}(I)$.

1.2. Partial elimination ideals. Now let $I$ be a homogeneous ideal in the graded ring $S=\mathbf{C}\left[a, x_{1}, x_{2}, \ldots, x_{r}\right]$ with maximal ideal $m$. I corresponds to a variety $X \subset \mathbf{P}^{r}$. We also write $\bar{S}=\mathbf{C}\left[x_{1}, x_{2}, \ldots, x_{r}\right]$ with maximal ideal $\bar{m}$.

For any $0 \neq F \in I$, there is a maximal $k$ for which we may write

$$
F=a^{k} f_{k}+a^{k-1} f_{k-1}+a^{k-2} f_{k-2}+\cdots+f_{0},
$$

with $f_{k} \neq 0$ and with each $f_{i} \in \bar{S}$. 
Definition 1.7. For fixed $k$, set $\mathrm{K}_{k}(I)=\left\{f_{k} \mid F \in I\right.$ as above $\}$. Then $\mathrm{K}_{k}(I)$ is the $\mathbf{k}^{\text {th }}$-partial elimination ideal of the ideal $I$. We will often write $\mathrm{K}_{k}$ if the ideal $I$ is understood. The sheafification of the $\mathrm{K}_{k}$ will be written $\mathcal{K}_{k}$.

Remark 1.8. Geometrically, the 1-elimination order corresponds to the projection $\pi: \mathbf{P}^{r} \rightarrow \mathbf{P}^{r-1}$ from $[1,0, \ldots, 0]$. The ideal $\mathrm{K}_{0}$ is the ideal of the projection; that is, it is the ideal of $\pi(X)$. We set $\pi(X)=\bar{X}$.

Definition 1.9. The Jacobi ideal of $I, J(I)$, is the ideal with generators $\left\{\partial F / \partial x_{i}\right.$ (and $\partial F / \partial a) \mid F \in I, \quad 1 \leq i \leq r\}$.

The important results we need about partial elimination ideals are collected in the following.

\section{Proposition 1.10.}

(1) Let $\pi$ be the projection of $X \subset \mathbf{P}^{r}$ from $[1,0,0, \ldots, 0]$ to $\mathbf{P}^{r-1}$. Then the $\mathrm{K}_{k}(I)$ are the ideals of $\left\{p \in \pi(X) \mid \operatorname{mult}_{p}(\pi(X))>k\right\}$.

(3) In general coordinates, the Jacobi ideal of $\mathrm{K}_{k-1}$ is contained in $\mathrm{K}_{k}$.

(3) The partial elimination ideal $\mathrm{K}_{0}$, the ideal of the projection, is saturated.

\section{Partial Elimination ideal SheaVes Bound REGUlarity}

2.1. A bound on regularity. Suppose $X \subset \mathbf{P}^{r}$ is a reduced, irreducible nondegenerate variety of degree $d$ and dimension $n$. Recent work on regularity has sought to achieve the conjectured bound [EG84:

$$
\operatorname{reg}(X) \leq d-(r-n)+1 .
$$

In dimensions $n=1,2$, the bound has been proven to hold; results are incomplete in higher dimensions (see [GLP83], [Laz87, Pin86 and see Kwa98 for a summary of known results). It is hoped that the following theorem may be of use in the pursuit of results on regularity in higher dimensions.

Theorem 2.1. Let $X$ be a reduced, irreducible, nondegenerate variety of degree $d$ and dimension $n$ in $\mathbf{P}^{r}$, defined by the ideal $I$, with sheafification $\mathcal{I}_{X}$. Let $\pi: \mathbf{P}^{r} \rightarrow$ $\mathbf{P}^{r-1}$ be a generic projection of $X$. Set

$$
i_{0}(X)=\max \left\{i \mid \mathcal{K}_{i} \text { defines a nonempty variety }\right\} .
$$

Then

$$
\operatorname{reg} \mathcal{I}_{\mathrm{X}} \leq \max _{i \leq i_{0}}\left\{\operatorname{reg} \mathcal{K}_{i}+i\right\}
$$

Proof of the Theorem. We will use generic initial ideal methods. Choose coordinates $\left[a, x_{1}, \ldots, x_{r}\right]$ for $\mathbf{P}^{r}$ so that $[1,0, \ldots, 0] \notin X$ and so that the projection from $[1,0, \ldots, 0]$ is generic, and corresponds to elimination of the variable $a$ from the ideal $I$. Let $\mathrm{K}_{i}$ be the partial elimination ideals, and let $\mathcal{K}_{i}$ be the sheafification of the $\mathrm{K}_{i}$. Fix as the monomial order the $\{1, r\}$-product order, which we also call the 1-elimination order. By the Bayer-Stillman Theorem, we know that

$$
\operatorname{reg} I \leq \max \{\operatorname{deg}(f) \mid f \text { is a generator of } \operatorname{gin}(I)\},
$$

where the gin is taken in any monomial order. Thus, for the 1-elimination order, we have

$$
\operatorname{gin}(I)=\left(a^{i} f_{i, 1}, a^{i} f_{i, 2}, \ldots, a^{i} f_{i, t_{i}}, a^{i-1} f_{i-1,1}, a^{i-1} f_{i-1,2}, \ldots, a^{i-1} f_{i-1, t_{i-1}}, \ldots,\right.
$$

$$
\left.a f_{1,1}, a f_{1,2}, \ldots, a f_{1, t_{1}}, f_{0,1}, \ldots, f_{0, t_{0}}\right) \text {, }
$$


where the $f_{i, j} \in \bar{S}=\mathbf{C}\left[x_{1}, \ldots, x_{r}\right]$, and, for fixed $k$, the elements of the set

$$
\left\{f_{k, j} \mid 1 \leq j \leq t_{k}\right\}
$$

generate the (not necessarily saturated) rlex $\operatorname{gin}\left(\mathrm{K}_{k}\right)$.

The maximal degree of a generator of $\operatorname{gin}(\mathrm{I})$ involving $a^{k}$ is thus equal to the maximal degree of a generator of $\operatorname{gin}\left(\mathrm{K}_{k}\right)$ plus $k$.

Claim 2.2. The generators of $\operatorname{gin}(I)$ of maximal degree do not involve $x_{r}$ to a positive exponent.

Once the claim is proved, we will have proved the theorem. To see this, let $f$ be a generator of $\operatorname{gin}(I)$ of maximal degree, say $\operatorname{deg}(f)=k+h$, where $f=a^{k} f_{k, j}$, and which does not involve $x_{r}$. We must have that $h$ is the regularity of $K_{k}$. Notice that the satiety of $\mathrm{K}_{k}$ is at most $h$, because all terms of $\operatorname{gin}\left(\mathrm{K}_{k}\right)$ are of degree at most $h$. The set in (3) is the set of rlex generators of gin $\left(\mathrm{K}_{k}\right)$, and so the maximal degree of a generator is equal to $\operatorname{reg}\left(\mathrm{K}_{k}\right)$, and then $\operatorname{reg}\left(\mathrm{K}_{k}\right)=\operatorname{reg}\left(\mathcal{K}_{k}\right)$.

We now prove Claim 2.2 by contradiction. Assume that the claim is false, that is, that some generator of maximal degree in $\operatorname{gin}(I)$ has the form

$$
a^{k} x_{0}^{h_{0}} \cdot \ldots \cdot x_{r}^{h_{r}}, \text { with } h_{r}>0
$$

Let $T$ be the unique reduced Gröbner basis with respect to the 1-elimination order for $I$. Let $P$ be an element of $T$ of maximal degree and involving $x_{r}$, say $\operatorname{deg}(P)=k+h$,

$$
P=a^{k} x_{1}^{h_{1}} x_{2}^{h_{2}} \cdot \ldots \cdot x_{r-1}^{h_{r-1}} x_{r}^{h_{r}}+p \in T,
$$

where $\sum h_{i}=h$ and $\operatorname{deg}(p)=k+h, h_{r}>0$.

Now $p \neq 0$, since a product of monomials cannot be a generator of the nondegenerate and irreducible ideal $I$. Furthermore, $x_{r}$ cannot divide $p$, because then it divides all of $P$, and since $I$ is irreducible and nondegenerate, we would have $\frac{1}{x_{r}} P \in I$. So $P$ would not be a minimal generator of $I$.

By the Borel-fixedness of gin $(I)$, we may perform the elementary move

$$
e_{r-1}(\operatorname{LeadTerm}(P))=a^{k} x_{1}^{h_{1}} \cdot x_{r-2}^{h_{r-2}} x_{r-1}^{h_{r-1}+1} x_{r}^{h_{r}-1} \in \operatorname{gin}(I) .
$$

So there is a $Q \in T$,

$$
Q=a^{b_{0}} x_{1}^{b_{1}} x_{2}^{b_{2}} \cdot \ldots \cdot x_{r-2}^{b_{r-2}} x_{r-1}^{b_{r-1}} x_{r}^{b_{r}}+q,
$$

with $b_{0} \leq k, b_{r-1}=h_{r-1}+1$ (if not, $\operatorname{LeadTerm}(Q)$ would divide $\operatorname{LeadTerm}(P)$, which is a generator of the $\operatorname{gin}(I)), b_{r}<h_{r}, \sum b_{i} \leq k+h, q \neq 0$.

Set

$$
\begin{aligned}
G_{1} & =x_{r-1} P-a^{k-b_{0}} x_{1}^{h_{1}-b_{1}} \cdot \ldots \cdot x_{r-2}^{h_{r-2}-b_{r-2}} x_{r}^{h_{r}-b_{r}} Q \\
& =x_{r-1} p-a^{k-b_{0}} x_{1}^{h_{1}-b_{1}} \cdot \ldots \cdot x_{r-2}^{h_{r-2}-b_{r-2}} x_{r}^{h_{r}-b_{r}} q .
\end{aligned}
$$

Notice that $G_{1} \in I, \operatorname{deg}\left(G_{1}\right)=k+h+1$. Notice that $G_{1} \neq 0$ because, by (5), that would imply that $x_{r}$ divides $P$. Set $g_{1}=\operatorname{LeadTerm}\left(G_{1}\right)$. We now examine candidates for the lead term of $G_{1}$, based on (5)). Notice that

$$
g_{1} \leq \max \left\{\operatorname{LeadTerm}\left(x_{r-1} P\right), \operatorname{LeadTerm}\left(a^{k-b_{0}} x_{1}^{h_{1}-b_{1}} \cdot \ldots \cdot x_{r-2}^{h_{r-2}-b_{r-2}} x_{r}^{h_{r}-b_{r}} Q\right)\right\} .
$$

Since the two lead terms on the right side of the above inequality are equal and, by the construction of $G_{1}$, cancel, the inequality is in fact strict. 
From $G_{1}$ we will inductively construct an infinite sequence $G_{i}$ of polynomials of degree $k+h+1$ with strictly decreasing leading terms, thus leading to a contradiction.

Now assume inductively that we have defined

$$
G_{i} \text {, with } \operatorname{deg}\left(G_{i}\right)=k+h+1, G_{i} \neq 0, \operatorname{LeadTerm}\left(G_{i}\right)=g_{i} .
$$

Let $F_{i} \in T$ be the Gröbner basis element that has lead term $f_{i} \in \operatorname{gin}(I)$ so that $f_{i}$ divides $g_{i}$. We examine the possible cases for $g_{i}$.

If $g_{i}$ appears as a monomial term of $x_{r-1} p$, then we have an equation

$$
f_{i} v=g_{i}=x_{r-1} \hat{p}
$$

where $\hat{p}$ is a monomial term of $p$. Now by assumption $\operatorname{deg}\left(f_{i}\right) \leq k+h$, and $\operatorname{deg}\left(g_{i}\right)=k+h+1$; so $\operatorname{deg}(v)>0$. Since $T$ is a reduced Gröbner basis, $f_{i}$ does not divide $\hat{p}$, but $\operatorname{deg}\left(f_{i}\right) \leq \operatorname{deg}(\hat{p})$ by assumption. Together with (7), we see then that

$$
\begin{array}{r}
\operatorname{deg}_{x_{r-1}}\left(f_{i}\right)=\operatorname{deg}_{x_{r-1}}(\hat{p})+1, \\
\operatorname{deg}_{x_{j}}\left(f_{i}\right) \leq \operatorname{deg}_{x_{j}}(\hat{p}) \text { for all } j, \\
\operatorname{deg}_{a}\left(f_{i}\right) \leq \operatorname{deg}_{a}(\hat{p}),
\end{array}
$$

and so

$$
\operatorname{deg}_{x_{j}}\left(f_{i}\right)<\operatorname{deg}_{x_{j}}(\hat{p}) \text { for some } j \neq r-1 \operatorname{ordeg}_{a}\left(f_{i}\right)<\operatorname{deg}_{a}(\hat{p}) .
$$

If, in (8),$j<r-1$, then by the Borel-fixedness of $\operatorname{gin}(I)$, a sequence of elementary moves produces

$$
f^{\prime}=e_{j}\left(e_{j+1}\left(\ldots\left(e_{r-2}\left(f_{i}\right)\right) \ldots\right)\right) \in \operatorname{gin}(I) .
$$

The elementary move sequence has the effect of replacing an $x_{r-1}$ with an $x_{j}$. If $\hat{f}=\operatorname{LeadTerm}(\hat{F}), \hat{F} \in T$, is a generator of $\operatorname{gin}(I)$ dividing $f^{\prime}$, then by referring back to (7) we see that $\hat{f}$ divides $\hat{p}$, contradicting the fact that $T$ is a reduced Gröbner basis. The case of $\operatorname{deg}_{a}\left(f_{i}\right)<\operatorname{deg}_{a}(\hat{p})$ is similar.

Thus, if (8) holds, it must be that $j=r$. Then $x_{r}$ divides $v$, say $x_{r} v_{i}=v$, and so we can define

$$
G_{i+1}=G_{i}-c_{i} x_{r} v_{i} F_{i},
$$

with $c_{i}$ a constant, chosen so that $\operatorname{in}\left(G_{i}\right)$ cancels with $\operatorname{in}\left(-c_{i} x_{r} v_{i} F_{i}\right) . G_{i+1}$ is nonzero since $x_{r}$ does not divide $P . G_{i+1}$ is still of degree $k+h+1$, and the lead term of $G_{i+1}$ is necessarily later in the elimination order than $G_{i}$.

If $g_{i}$ does not appear as a term of $x_{r-1} p$, then $g_{i}$ is a term of

$$
a^{k-b_{0}} x_{1}^{h_{1}-b_{1}} \cdot \ldots \cdot x_{r-2}^{h_{r-2}-b_{r-2}} x_{r}^{h_{r}-b_{r}} q-x_{r} v_{i-1} F_{i-1},
$$

and we have

$$
f_{i} v=g_{i}=x_{r-1} \hat{q}
$$

where (since by assumption $\left.\operatorname{deg}\left(f_{i}\right) \leq k+h\right) \operatorname{deg}(v)>0$, and $\hat{q}$ is some term of (11). If $x_{r}$ divides $v$, then we again write $v=x_{r} v_{i}$, and define $G_{i+1}$ as in (10). If $x_{r}$ does not divide $v$, then we use Borel-fixedness of $\operatorname{gin}(I)$ and a sequence of elementary moves to produce

$$
f^{\prime}=e_{j}\left(e_{j+1}\left(\ldots\left(e_{r-1}\left(f_{i}\right)\right) \ldots\right)\right) \in \operatorname{gin}(I),
$$


which has the effect of replacing an $x_{r}$ with an $x_{j}$. There is then an $\hat{F} \in T$ that has lead term $\hat{f}$ dividing $f^{\prime}$, so that, returning to (12) , we have $\hat{f} x_{r} \hat{v}_{i}=g_{i}$. Redefine $F_{i}=\hat{F}$ and define $G_{i+1}$ as in (10). Again, $G_{i+1} \neq 0, G_{i+1}$ is still of degree $k+h+1$, and the lead term of $G_{i+1}$ is necessarily later in the elimination order than $G_{i}$.

Thus our induction produces an infinite sequence of $G_{i}$ with successively later lead terms (the degree of the $G_{i}$ stays fixed for all $i$ ), but all the $G_{i}$ are nonzero. This is the desired contradiction.

Thus it cannot be that any terms of gin(I) of maximal degree involve $x_{r}$. So terms of maximal degree do not involve $x_{r}$. Hence we have proved the claim and the theorem.

Note 2.3. If $X$ is smooth, there is an approach to possibly improving Theorem 2.1 yielding the bound

$$
\operatorname{reg}\left(\mathcal{I}_{X}\right) \leq \max \left\{\operatorname{reg}\left(\mathcal{K}_{0}\right), \operatorname{reg}\left(\mathcal{K}_{1}\right)+1\right\}
$$

The exact sequence

$$
0 \rightarrow \mathcal{K}_{0} \rightarrow \pi_{*}\left(\mathcal{I}_{X}\right) \rightarrow \mathcal{K}_{1}(-1) \rightarrow 0
$$

may be able to give us an immediate improvement on Theorem [2.1. Let

$$
\operatorname{reg}\left(\mathcal{K}_{0}\right)=m, \quad \operatorname{reg}\left(\mathcal{K}_{1}\right)=n, \quad p=\max \{m, n+1\} .
$$

Part of the associated cohomological long exact sequence of (14),

$$
\ldots \rightarrow H^{i}\left(\mathcal{K}_{0}(p-i)\right) \rightarrow H^{i}\left(\pi_{*} \mathcal{I}_{X}(p)\right) \rightarrow H^{i}\left(\mathcal{K}_{1}(p-i-1)\right) \rightarrow \ldots
$$

is exact for each $i>0$. By the choice of $p$, both the cohomology group on the left and the one on the right vanish; hence so does the one in the middle. Thus

$$
\left.\operatorname{reg}\left(\pi_{*}\left(\mathcal{I}_{X}\right)\right) \leq p \leq \max \left\{\operatorname{reg}\left(\mathcal{K}_{0}\right), \operatorname{reg}\left(\mathcal{K}_{1}\right)+1\right)\right\} .
$$

The aim is then to use this to prove

$$
\left.\operatorname{reg}\left(\mathcal{I}_{X}\right) \leq \max \left\{\operatorname{reg}\left(\mathcal{K}_{0}\right), \operatorname{reg}\left(\mathcal{K}_{1}\right)+1\right)\right\}
$$

To do this, one may look at the exact sequence

$$
0 \rightarrow \pi_{*} \mathcal{I}_{X} \rightarrow \pi_{*} \mathcal{O}_{\mathbf{P}^{r}} \rightarrow \pi_{*} \mathcal{O}_{X} \rightarrow R^{1} \pi_{*} \mathcal{I}_{X} \rightarrow R^{1} \pi_{*} \mathcal{O}_{\mathbf{P}^{r}} \rightarrow R^{1} \pi_{*} \mathcal{O}_{X} \rightarrow \ldots
$$

If $R^{1} \pi_{*} \mathcal{I}_{X}$ vanishes, a spectral sequence argument shows that the regularity of $\pi_{*} \mathcal{I}_{X}$ matches with the regularity of $\mathcal{I}_{X}$.

Remark 2.4. While this bound is no different than the theorem if $X$ is a curve, in higher dimensions the partial elimination ideals $\mathcal{K}_{k}, k \geq 2$, of the generic projections usually define nonempty varieties.

\section{Partial elimination ideals and the CANONiCAL BUndle}

In this section we use the partial elimination ideal sheaves to compute the canonical bundle of a variety as well as the canonical bundles of the multiple point loci, under the right conditions. This information is used to bound the regularity of the partial elimination ideal sheaves and, in conjunction with Theorem 2.1 to obtain regularity bounds for curves $X$. Many of the questions surrounding partial elimination ideals remain unanswered, and in particular we are limited by what is known about the singularities of a generic projection. 
For low-dimensional varieties where the Regularity Conjecture bound has been established, or something near to it, our methods will not provide any new information. However, these methods may shed some light on the possibility of near $\operatorname{deg}(X)$-regularity in arbitrary dimension. This chapter will hopefully pique interest in exploring the elimination order, partial elimination ideals, and their geometry.

3.1. Curves. Much effort has been made to understand multiple point loci. It has not always been clear what sheaf structure should be accorded to the loci. This is a broad subject unto itself, and it is beyond the scope of this paper to give even a thorough list of references, but see for instance the survey of Kleiman ([Kle82]).

We establish here the fact that for a generic projection of a space curve of degree $d$ into $\mathbf{P}^{2}$, the canonical bundle can be computed as a twist of $\mathcal{K}_{1} . d$-Regularity for curves follows by examining some cohomological exact sequences by using the Kodaira Vanishing Theorem and Theorem 2.1 and its corollary.

Let $X_{0} \subset \mathbf{P}^{r}$ be a nondegenerate smooth reduced irreducible curve of degree $d$. Let $\pi_{0}: \mathbf{P}^{r} \rightarrow \mathbf{P}^{3}$ be a generic projection, with $X_{0}$ isomorphic to its image, which we call $X$. Let $\pi: \mathbf{P}^{3} \rightarrow \mathbf{P}^{2}$ be a generic projection, so that the only singularities of $\pi$ are ordinary double points, and write $\pi(X)=\bar{X}$. Let $\mathcal{K}_{1}$ be the first partial elimination ideal of the map $\pi$. Let $\bar{D}_{2}$ be the variety defined by $\mathcal{K}_{1}$. By Proposition 1.10, $\bar{D}_{2}$ is also the set-theoretic double point locus.

We aim to prove

Theorem 3.1. The curve $X_{0}$ is d-regular.

Remark 3.2. This is a weaker bound for curves than what is already known.

Proof. We will first compute the canonical bundle. Following the method of [Xu94, we consider the blowup

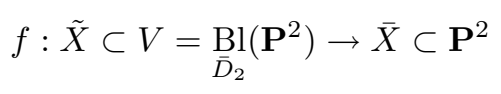

along $\bar{D}_{2}$, with exceptional divisor $E=\sum_{q_{i} \in \bar{D}_{2}} E_{i}$. This gives us

$$
\begin{aligned}
K_{\tilde{X}} & =\left.\left(K_{V}+\tilde{X}\right)\right|_{\tilde{X}} \\
& =\left.\left(f^{*}\left(K_{\mathbf{P}^{2}}\right)+E\right)\right|_{\tilde{X}}+\left.\left(f^{*}(\bar{X})-2 E\right)\right|_{\tilde{X}} \\
& =\left.\left(f^{*}\left(K_{\mathbf{P}^{2}}+\bar{X}\right)-E\right)\right|_{\tilde{X}} \\
& =\left.\left(f^{*}\left(\mathcal{O}_{\bar{X}}(d-3)\right)-E\right)\right|_{\tilde{X}},
\end{aligned}
$$

where $\mathcal{O}_{\bar{X}}=\mathcal{O}_{\mathbf{P}^{2}} / \mathcal{K}_{0}$. This computation enables us to prove

Lemma 3.3. $K_{\tilde{X}} \cong \mathcal{K}_{1} \otimes \mathcal{O}_{\bar{X}}(d-3)$.

Proof of the Lemma. Suppose $g$ is a section of $H^{0}\left(\mathcal{K}_{1} \otimes \mathcal{O}_{\bar{X}}(d-3)\right)$. Then since $g$ vanishes on $\bar{D}_{2}$, it induces a section of $K_{\tilde{X}}$.

Conversely, fix $q_{i_{0}} \in \bar{D}_{2}$, and an open neighborhood $U$ of $q_{i_{0}}$ not meeting $\bar{D}_{2}$ except at $q_{i_{0}}$. Take local analytic coordinates $\left(u_{1}, u_{2}\right)$ on $U$ with $q_{i_{0}}=(0,0), \bar{X}=$ $\left\{u_{1} u_{2}=0\right\}$, and hence so that the local sections of $\mathcal{K}_{1}$ are $u_{1}$ and $u_{2}$. Consequently, the blowup is defined locally near the exceptional divisor $E_{i_{0}}$ as a subset of $\mathbf{A}^{2} \times \mathbf{P}^{1}$ by coordinates $\left(\left(u_{1}, u_{2}\right),\left[R_{1}, R_{2}\right]\right)$ by $u_{1} R_{2}=u_{2} R_{1}$. $\tilde{X}$ is given by $\left\{u_{1}=R_{1}=0\right\}$, which has local coordinate $u_{2}$, and $\left\{u_{2}=R_{2}=0\right\}$, which has local coordinate $u_{1}$. 
From the equation defining $\bar{X}$ down in $U$, we get the relation $u_{1} d u_{2}+u_{2} d u_{1}=0$. Hence an element of the canonical bundle of $\tilde{X}$ takes the form

$$
\frac{r d u_{1}}{u_{1}}=-\frac{r d u_{2}}{u_{2}}
$$

where $r$ must be of degree $d-3$ since, recalling that $\bar{X}$ is a hypersurface of degree $d$ in $\mathbf{P}^{2}$, the differential $d u_{1} / u_{1}=d u_{2} / u_{2}$ has a pole of degree $d-3$. Thus, $r \in$ $\mathcal{O}_{f^{-1}(U) \cap X}(d-3)$. Now

$$
\begin{aligned}
\mathcal{O}_{f^{-1}(U) \cap X} & =\bigoplus_{i=1,2}\left(u_{3-i}\right) \mathcal{O}_{f^{-1}(U)} /\left(u_{i}\right) \\
& \cong\left(u_{1}, u_{2}\right) \mathcal{O}_{U} /\left(u_{1} u_{2}\right) \\
& \cong \mathcal{K}_{1} \otimes \mathcal{O}_{\bar{X} \cap U} .
\end{aligned}
$$

Hence $r$ corresponds to a section of $\mathcal{K}_{1} \otimes \mathcal{O}_{\bar{X}}(d-3)$. This proves the lemma.

In order to prove that $\mathcal{I}_{X_{0}}$ is $d$-regular, we will show that $\mathcal{I}_{X}$ is $d$-regular. Then, since the map $\pi_{0}$ is an isomorphism and a generic projection, it can be decomposed into a sequence of maps $\pi_{i}{ }^{\prime}: \mathbf{P}^{r-i} \rightarrow \mathbf{P}^{r-i-1}$ which are generic projections and in which $X_{0}$ is isomorphic to its succesive images under the $\pi_{i}{ }^{\prime}$. Then, by Theorem 2.1, we have

$$
\operatorname{reg}\left(X_{0}\right) \leq \operatorname{reg}(X) .
$$

In order to prove that $\mathcal{I}_{X}$ is $d$-regular, we will show that $\mathcal{K}_{0}$ is $d$-regular and $\mathcal{K}_{1}$ is $(d-1)$-regular. Then, since $\pi$ has at most double points, so that the $\mathcal{K}_{k}, k \geq 2$, define empty varieties, Theorem 2.1 now gives us

$$
\begin{aligned}
\operatorname{reg}\left(\mathcal{I}_{X}\right) & \leq \max \left\{\operatorname{reg}\left(\mathcal{K}_{0}\right), \operatorname{reg}\left(\mathcal{K}_{1}\right)+1\right\} \\
& \leq \max \{d,(d-1)+1\} \\
& \leq d
\end{aligned}
$$

Since $\bar{X}$ is an irreducible hypersurface of degree $d, \mathcal{K}_{0}$ is generated by a single equation in degree $d$, i.e., $\mathcal{K}_{0} \cong \mathcal{O}_{\mathbf{P}^{2}}(-d)$. Also, $\mathcal{O}_{\mathbf{P}^{2}}(-3)=K_{\mathbf{P}^{2}}$. We now utilize the exact sequence

$$
0 \rightarrow \mathcal{K}_{0} \rightarrow \mathcal{K}_{1} \rightarrow \mathcal{K}_{1} / \mathcal{K}_{0} \rightarrow 0
$$

Recalling the lemma, we know that $\mathcal{K}_{1} / \mathcal{K}_{0} \cong K_{\bar{X}}(-(d-3))$. Hence, if we twist (16) by $(d-3)$ and take the cohomological exact sequence, we get, in part,

$$
\cdots \rightarrow H^{1}\left(K_{\bar{X}}\right) \rightarrow H^{2}\left(K_{\mathbf{P}^{2}}\right) \rightarrow H^{2}\left(\mathcal{K}_{1}(d-3)\right) \rightarrow H^{2}\left(K_{\bar{X}}\right) \rightarrow \cdots .
$$

Now the map $H^{1}\left(K_{\bar{X}}\right) \rightarrow H^{2}\left(K_{\mathbf{P}^{2}}\right)$ is the Gysin map, and is an isomorphism in this case. Also, $H^{2}\left(K_{\bar{X}}\right)=0$ because $X$ is a curve. Thus $H^{2}\left(\mathcal{K}_{1}(d-2)\right)=0$. By twisting instead by $(d-2)$, we have

$$
\cdots \rightarrow H^{1}\left(K_{\mathbf{P}^{2}}(1)\right) \rightarrow H^{1}\left(\mathcal{K}_{1}(d-2)\right) \rightarrow H^{1}\left(K_{\bar{X}}(1)\right) \rightarrow \cdots .
$$

Here the Kodaira Vanishing Theorem gives us that the cohomology groups on the left and right are both zero; hence $H^{1}\left(\mathcal{K}_{1}(d-2)\right)=0$. So $\mathcal{K}_{1}$ is $(d-1)$-regular. Since $\mathcal{K}_{0}$ is generated in degree $d$, it is $d$-regular. This proves Theorem 3.1 
3.2. Higher dimensions. The canonical bundle of the image of a generic projection can be computed as a twist of the ideal sheaf $\mathcal{K}_{1}$ under some mild assumptions.

We need to expand a definition: Let $X$ be a smooth, nondegenerate, reduced, irreducible variety of dimension $n$ and degree $d$ in $\mathbf{P}^{r}$. Take generic coordinates $a_{1}, a_{2}, \ldots, a_{r-s}, x_{0}, x_{1}, \ldots, x_{s}$ on $\mathbf{P}^{r}$ so that $\Lambda=\left\{x_{0}=0, x_{1}=0, \ldots, x_{s}=0\right\}$ does not meet $X$ and so that projection from $\Lambda$ is generic. Fix $\pi: \mathbf{P}^{r} \rightarrow \mathbf{P}^{s}$ as the projection from $\Lambda$. Let $\bar{X}=\pi(X)$. Fix as the monomial order on $\mathbf{P}^{r}$ the $(r-s)$-elimination order on the variables $a_{1}, a_{2}, \ldots, a_{r-s}, x_{0}, x_{1}, \ldots, x_{s}$. This elimination corresponds to the projection of $\mathbf{P}^{r}$ from $\Lambda$ to $\mathbf{P}^{s}$. The coordinates on $\mathbf{P}^{s}$ will then be $x_{0}, x_{1}, \ldots, x_{s}$. Let $X$ be defined by the saturated ideal $I \subset$ $\mathbf{C}\left[a_{1}, a_{2}, \ldots, a_{r-s}, x_{0}, x_{1}, \ldots, x_{s}\right]$ with sheafification $\mathcal{I}$.

Definition 3.4. The $(1, r-s)$-th partial elimination ideal of $I$ is defined as

$$
\mathrm{K}_{1}(I)=\left\{f \mid a_{r-s} f+g \in I \text { with } f, g \in \mathbf{C}\left[x_{0}, x_{1}, \ldots, x_{s}\right]\right\} .
$$

Remark 3.5. If we decompose the map $\pi$ into generic projections $\pi_{i}{ }^{\prime}: \mathbf{P}^{r-i} \rightarrow$ $\mathbf{P}^{r-i-1}$, the $(1, r-s)$-elimination ideal will be identical to the first partial elimination ideal of the projection $\pi_{r-s+1}{ }^{\prime}$.

Now fix $s=n+1$.

We now prove

Proposition 3.6. Let $X$ be a smooth, irreducible, reduced, and nondegenerate variety in $\mathbf{P}^{r}$. Let $S$ be the set of singularities of the generic projection of $X$ to $\mathbf{P}^{n+1}$ other than the ordinary double points. Assume $S$ is codimension 2 in $X$. Then

$$
\mathrm{K}_{\bar{X}} \cong \mathcal{K}_{1} \otimes \mathcal{O}_{\overline{\mathrm{X}}}(d-n-2) .
$$

Proof. Let $\bar{D}_{2}$ be the variety defined by $\mathcal{K}_{1}$. By blowing up along $\bar{D}_{2}$, and computing the canonical bundle of $\bar{X}$ on the blowup, a computation similar to Lemma 3.3 shows that the canonical bundle $\bar{X}$ is isomorphic to $\mathcal{K}_{1} \otimes \mathcal{O}_{\overline{\mathrm{X}}}(d-n-2)$ away from $S$. Since $S$ is codimension 2 in $X$, there is a unique analytic extension so that the sheaves match globally. This proves the proposition.

Corollary 3.7. Let $X$ be a smooth, irreducible, reduced, nondegenerate variety in $\mathbf{P}^{r}$. If $X$ is an $n$-dimensional variety in $\mathbf{P}^{n+2}$, and the set $S$, defined in the proposition, of the generic projection of $X$ to $\mathbf{P}^{n+1}$ has codimension 2 in $\bar{X}$, then $\mathcal{K}_{1}$ is $(d-1)$-regular.

Proof. In the situation of the generic projection $\pi: \mathbf{P}^{n+2} \rightarrow \mathbf{P}^{n+1}$, we can compute a regularity bound for $\mathcal{K}_{1}$. We have an exact sequence

$$
0 \rightarrow \mathcal{K}_{0} \rightarrow \mathcal{K}_{1} \rightarrow \mathcal{K}_{1} / \mathcal{K}_{0} \rightarrow 0
$$

Since $\bar{X}$ is irreducible and codimension 1 in $\mathbf{P}^{n+1}, \mathcal{K}_{0} \cong \mathcal{O}_{\mathbf{P}^{n+1}}(-d)$. So, in conjunction with Proposition 3.6 (17) twisted by $d-n-2$ gives us

$$
0 \rightarrow K_{\mathbf{P}^{n+1}} \rightarrow \mathcal{K}_{1}(d-n-2) \rightarrow K_{\bar{X}} \rightarrow 0 .
$$

Various twists of segments of the cohomological long exact sequence of (18) give us

$$
\begin{aligned}
\cdots & \rightarrow H^{i}\left(K_{\mathbf{P}^{n+1}}(n+1-i)\right) \rightarrow H^{i}\left(\mathcal{K}_{1}(d-1-i)\right) \rightarrow H^{i}\left(K_{\bar{X}}(n+1-i)\right) \\
& \rightarrow \cdots .
\end{aligned}
$$


By the Kodaira Vanishing Theorem, we have

$$
H^{i}\left(K_{\bar{X}}(m)\right)=0 \text { and } H^{i}\left(K_{\mathbf{P}^{n+1}}(m)\right)=0
$$

for all $i>0, m>0$. Moreover, as a sheaf on $\mathbf{P}^{n+1}, H^{i}\left(\mathcal{K}_{1}(m)\right)=0$ for all $i>n+1$ and all $m$. Thus we have

$$
H^{i}\left(\mathcal{K}_{1}(d-1-i)\right)=0 \text { for } 0<i<n+1 .
$$

It remains to show that $H^{n+1}\left(\mathcal{K}_{1}(d-n-2)\right)=0$. For this we examine part of the cohomological exact sequence as follows:

$$
\cdots \rightarrow H^{n}\left(K_{\bar{X}}\right) \rightarrow H^{n+1}\left(K_{\mathbf{P}^{n+1}}\right) \rightarrow H^{n+1}\left(\mathcal{K}_{1}(d-n-2)\right) \rightarrow H^{n+1}\left(K_{\bar{X}}\right) \rightarrow \cdots .
$$

Here, the Gysin map $H^{n}\left(K_{\bar{X}}\right) \rightarrow H^{n+1}\left(K_{\mathbf{P}^{n+1}}\right)$ is an isomorphism, and since $X$ is $n$-dimensional, $H^{n+1}\left(K_{\bar{X}}\right)=0$. Thus $H^{n+1}\left(\mathcal{K}_{1}(d-n-2)\right)$ also vanishes. So $\mathcal{K}_{1}$ is $(d-1)$-regular. This proves the corollary.

\section{The REgularity of HigheR-DIMEnsional VARIETIES AND THE SHARP REGULARITY BOUND}

Ideally, one hopes to prove that each successive $k$-fold locus can be computed as a twist or sum of twists of $\mathcal{K}_{k}$, and then to use this to bound the regularity of the $\mathcal{K}_{k}$. However, this involves discerning the types of singularities beyond ordinary $k$-fold points and reconciling the behavior of the canonical bundle there with its behavior near ordinary multiple points. With this information, the next obstacle is to use Kodaira Vanishing or other geometric or cohomological tools to get the regularity of the $\mathcal{K}_{k}$. From there, an application of Theorem 2.1 would yield a bound on the regularity of $\mathcal{I}_{X}$.

To improve Theorem 2.1, note that while the maximum degree of a generator of the reverse lexicographic gin gives the exact regularity of the ideal, the elimination order may not. The examples seem to indicate that for each generic projection, we have $\operatorname{reg}\left(\mathcal{I}_{X}\right)<\operatorname{reg}\left(\mathcal{K}_{0}\right)$. Using this, a smooth nondegenerate projective variety $X \subset \mathbf{P}^{r}$ of dimension $n$ and degree $d$ could be projected generically to $\bar{X} \subset \mathbf{P}^{n+1}$, which is cut out by a single equation of degree $d$, and so $\operatorname{reg}(\bar{X})=d$. Since we have a chain of $r-n-1$ strict inequalities between the regularity of $X$ and the regularity of $\bar{X}$, we get that

$$
\operatorname{reg}\left(\mathcal{I}_{X}\right) \leq \operatorname{reg}\left(\mathcal{I}_{\bar{X}}\right)-(r-n-1)=d-r+n+1,
$$

which is the conjectured bound.

\section{REFERENCES}

[BS87] David Bayer and Michael Stillman, A criterion for detecting $m$-regularity, Invent. Math. 87 (1987), no. 1, 1-11. MR 87k:13019

[EG84] David Eisenbud and Shiro Goto, Linear free resolutions and minimal multiplicity, J. Algebra 88 (1984), no. 1, 89-133. MR 85f:13023

[GLP83] L. Gruson, R. Lazarsfeld, and C. Peskine, On a theorem of Castelnuovo, and the equations defining space curves, Invent. Math. 72 (1983), no. 3, 491-506. MR 85g:14033

[Gre98] Mark Green, Generic initial ideals, Six Lectures On Commutative Algebra (Elias, 1996) (J. Elias, J. M. Giral, R. M. Miró-Roig, and S. Zarzuela, eds.), Birkhäuser-Verlag, Basel, 1998, Papers from the Summer School on Commutative Algebra held in Bellaterra, July 16-26, 1996, pp. 119-186. MR 99m:13040

[Kle82] Steven L. Kleiman, Multiple point formulas for maps, Enumerative geometry and classical algebraic geometry (Nice, 1981), Birkhäuser Boston, Mass., 1982, pp. 237-252. MR 84j:14059 
REGULARITY, PARTIAL ELIMINATION IDEALS AND CANONICAL BUNDLE

[Kwa98] Sijong Kwak, Castelnuovo regularity for smooth subvarieties of dimensions 3 and 4, J. Algebraic Geom. 7 (1998), no. 1, 195-206. MR 2000d:14043

[Laz87] Robert Lazarsfeld, A sharp Castelnuovo bound for smooth surfaces, Duke Math. J. 55 (1987), no. 2, 423-429. MR 89d:14007

[Pin86] Henry C. Pinkham, A Castelnuovo bound for smooth surfaces, Invent. Math. 83 (1986), no. 2, 321-332. MR 87c:14044

[Xu94] Geng Xu, Subvarieties of general hypersurfaces in projective space, J. Differential Geom. 39 (1994), no. 1, 139-172. MR 95d:14043

Department of Mathematics, California State University, Dominguez Hills, 1000 E. Victoria St., Carson, California 90747

E-mail address: mjones@csudh.edu 